\title{
A NMR Study of Solute-Solvent Interactions as a Function of the Nitrogen Shielding of Pyridine $\mathbf{N}$-Oxide
}

\author{
M. WitANOWSKI, W. SicinSKA, AND S. BIERNAT \\ Institute of Organic Chemistry, Polish Academy of Sciences, 01-224 Warsaw, Poland \\ AND \\ G. A. WEBB* \\ Department of Chemistry, University of Surrey, Guildford, Surrey, United Kingdom
}

Received November 1, 1988

\begin{abstract}
The range of solvent effects on the nitrogen shielding of pyridine $\mathrm{N}$-oxide is found to be comparable to that of pyridine, amounting to about $30 \mathrm{ppm}$. The largest contribution to this range arises from hydrogen bonding from the solvents to the oxygen atom of pyridine $\mathrm{N}$-oxide. For the first time it is demonstrated that hydrogen bonding to a neighboring atom can have a comparable effect on nitrogen shielding as would direct hydrogen bonding to the nitrogen atom. Medium polarity effects on the nitrogen shielding of pyridine and its $N$-oxide are of the same sign and order of magnitude. This is corroborated by INDO/S-SOS shielding calculations using the solvaton model. These medium polarity effects amount to a nitrogen shielding variation not exceeding $6 \mathrm{ppm}$ while those due to hydrogen bonding exceed $20 \mathrm{ppm}$. @ 1989 Academic Press, Inc.
\end{abstract}

We have previously shown that solute-solvent interactions have a significant effect on the nuclear shieldings of $s p^{2}$ type nitrogen atoms in various molecules $(1-6)$. It is thus clear that nitrogen NMR is a very suitable tool for investigating the nature of solute-solvent interactions. The range of shieldings for such nitrogen atoms, produced by a change in solvent, can be as large as $40 \mathrm{ppm}$. The largest shielding variations occur when the lone pair is involved in $s p^{2}$ type hybridization and is available for hydrogen bonding with protic solvents. Such hydrogen bonding effects can produce an increase of nitrogen shiclding in cxcess of $20 \mathrm{ppm}$ such as in pyridine and alkyl nitrites (5).

If the nitrogen lone pair is not available for hydrogen bonding purposes, as in the nitro moiety of nitromethane $(4,5)$ and methyl nitrate $(6)$, then hydrogen bonding may occur via the oxygen lone pair electrons. In this case the contribution of hydrogen bonding to a change of solvent, on the nitrogen shielding, does not exceed $2 \mathrm{ppm}$. It is thus of interest to compare the solvent effects on the nitrogen shielding of pyridine with that of pyridine $N$-oxide, where hydrogen bonding with the solvent may only occur with the oxygen lone pair electrons as conventionally indicated

\footnotetext{
* To whom correspondence should be addressed.
} 


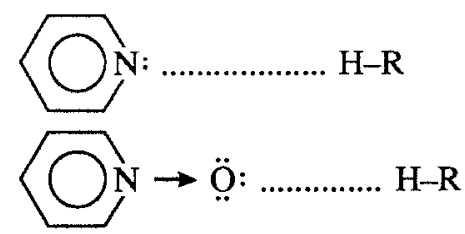

The various solvent contributions to the variation of nitrogen nuclear shieldings may be separated by means of a linear four-parameter model which incorporates solvent properties such as hydrogen bond donor and acceptor strengths as well as polarizability-polarity interactions $(5,6)$. The effects of the medium polarity on nitrogen shielding can then be compared with those for nonspecific solute-solvent interactions obtainable from the solvaton model (4-8). To date we have found a good agreement between these two approaches $(5,6)$.

In general large shielding effects, arising from the use of hydrogen bonding solvents, are interpreted in terms of a direct interaction between the nitrogen lone pair and the solvent $(1,2)$. It is thus of interest to determine whether the presence of an oxygen atom in pyridine $N$-oxide will result in a weaker influence of the solvent on nitrogen nuclear shielding. Consequently we hope to investigate whether a large nitrogen solvent shift necessarily implies a direct solute-solvent interaction involving the solute nitrogen atom.

\section{RESULTS AND DISCUSSION}

High-precision ${ }^{14} \mathrm{~N}$ NMR measurements have been made using a high magnetic field, a lineshape fitting procedure, and a rigorous calibration technique as described under Experimental. In Table 1 we report the nitrogen shielding of pyridine $N$-oxide taken on solutions in 13 solvents which cover a range of hydrogen bonding properties and differences in polarity. Inspection of the data shows that the nitrogen shielding of pyridine $\mathrm{N}$-oxide spans a range in excess of $30 \mathrm{ppm}$ for the chosen set of solvents. This compares with a nitrogen shielding range of about $40 \mathrm{ppm}$ for pyridine in a similar series of solvents. However, the range of $30 \mathrm{ppm}$ for pyridine $N$-oxide implies that hydrogen bonding effects for this molecule are large. Nevertheless one cannot exclude the possibility of significant polarity effects to this observed shielding range since pyridine $N$-oxide is a highly polar molecule.

In order to estimate the various contributions to the nitrogen shielding arising from hydrogen bonding effects, on the one hand, and nonspecific polarity-polarization effects, on the other, we employ the four-parameter master equation $(9)$,

$$
X Y Z=X Y Z_{0}+S\left(\pi^{*}+d \delta\right)+a \alpha+b \beta,
$$

where $X Y Z$ is a chosen molecular property such as nitrogen shielding for a solute molecule in a particular solvent; $X Y Z_{0}$ is the molecular property in a given reference state, e.g., cyclohexane solution; $\pi^{*}$ is the polarizability-polarity term for the solvent; $\alpha$ represents its hydrogen bond donor strength; $\beta$ represents its hydrogen bond acceptor strength; $\delta$ is a correction for polychlorinated solvents $(\delta=0.5)$ and aromatic solvents $(\delta=1.0)$; and $S, d, a$, and $b$ are the corresponding responses of the appropriate solute molecular property to the relevant solvent property. The solvent parameters employed in the present work are presented in Tablc 2 . The values used are based 
TABLE 1

${ }^{14}$ N NMR Data for Pyridine $N$-Oxide in Various Solvents

\begin{tabular}{|c|c|c|}
\hline Solvent & ${ }^{14} \mathrm{~N}_{\text {Shielding }}{ }^{a}$ (ppm) & $\begin{array}{c}\text { Half-height }{ }^{b} \\
\text { width of }{ }^{14} \mathrm{~N} \text { signal (hertz) }\end{array}$ \\
\hline Cyclohexane $(0.008 M)^{c}$ & 76.47 & 9.3 \\
\hline $\mathrm{Et}_{2} \mathrm{O}(0.064 M)^{c, d}$ & 78.78 & 15.2 \\
\hline $\mathrm{CCl}_{4}(0.128 M)^{c}$ & 80.84 & 26.3 \\
\hline Benzene & 81.36 & 20.2 \\
\hline Dioxane & 81.40 & 27.2 \\
\hline Acetone & 82.85 & 20.2 \\
\hline DMSO & 85.64 & 55.5 \\
\hline $\mathrm{CH}_{2} \mathrm{Cl}_{2}$ & 85.66 & 28.2 \\
\hline $\mathrm{CHCl}_{3}$ & 86.78 & 53.8 \\
\hline EtOH & 97.06 & 130.2 \\
\hline $\mathrm{MeOH}$ & 97.53 & 71.7 \\
\hline $\mathrm{H}_{2} \mathrm{O}$ & 106.47 & 102.0 \\
\hline $\mathrm{CF}_{3} \mathrm{CH}_{2} \mathrm{OH}$ & 106.61 & 386.3 \\
\hline
\end{tabular}

Note. The results are for $0.25 \mathrm{M}$ solutions at $35 \pm 0.2^{\circ} \mathrm{C}$.

${ }^{a} \Lambda$ ll shieldings are reported with respect to neat nitromethane such that an increase in shielding corresponds to a positive increment. The data given are corrected for solvent magnetic susceptibility effects and are accurate to within 0.1 ppm.

${ }^{b}$ Linewidth data are accurate to between 1.0 and $0.3 \mathrm{~Hz}$ as described under Experimental.

${ }^{c}$ Saturated solution used which is less than $0.25 \mathrm{M}$.

${ }^{d}$ Temperature of experiment is $30 \pm 0.2^{\circ} \mathrm{C}$.

upon our earlier work in this field $(5,6)$ which have provided successful analyses of the various effects of solvents on nitrogen nuclear shielding. As shown in Table 2 the choice of solvents used covers a wide range of hydrogen bonding and polarizabilitypolarity properties. Dielectric constant data for the solvents are also included in Table 2.

In Table 3 are given the results of a least-squares fitting of the observed nitrogen shieldings of pyridine $N$-oxide in a range of solvents to those predicted by Eq. [1] . The data given in Table 3 do not include those for aqueous solutions of pyridine $N$ oxide. If the results for water are included in Eq. [1] a serious decrease in the correlation coefficient is observed. This is very probably due to some uncertainty in the parameters for water given in Table 2. The results in Table 3 may be compared with those obtained in a similar manner for pyridine (5), where the nitrogen shielding varies from $57.7 \mathrm{ppm}$ for a cyclohexane solution to $96.1 \mathrm{ppm}$ for a solution in $\mathrm{CF}_{3}$ $\mathrm{CH}_{2} \mathrm{OH}$. An analogous fitting to Eq. [1] yields $X Y Z_{0}=57.89 \pm 0.87 \mathrm{ppm}, a=21.50$ $\pm 0.97 \mathrm{ppm}, b=-0.35 \pm 1.33 \mathrm{ppm}, S=4.88 \pm 1.48 \mathrm{ppm}$, and $d=0.002 \pm 0.23$ for pyridine. In general the nitrogen shieldings of pyridine $N$-oxide in various solvents are 10 to $20 \mathrm{ppm}$ higher than those for pyridine in a given solvent. This is in agreement with general observations concerning nitrogen shieldings of $s p^{2}$ hybridized nitrogen atoms upon involvement of the nitrogen lone pair electrons in bonding (11).

Our present results show that the effect on the nitrogen shielding of hydrogen bond- 
TABLE 2

Solvent Parameters Employed

\begin{tabular}{|c|c|c|c|c|c|}
\hline \multirow[b]{2}{*}{ Solvent } & \multicolumn{4}{|c|}{ Parameters used in Eq. [1] } & \multirow{2}{*}{$\begin{array}{l}\text { Dielectric } \\
\text { constant }^{a}\end{array}$} \\
\hline & $\alpha$ & $\beta^{b}$ & $\pi^{*}$ & $\delta$ & \\
\hline Cyclohexane & 0.0 & 0.0 & 0.0 & 0.0 & 1.87 \\
\hline $\mathrm{CCl}_{4}$ & 0.0 & 0.0 & 0.29 & 0.5 & 2.21 \\
\hline Benzene & 0.0 & 0.0 & 0.59 & 1.0 & 2.25 \\
\hline $\mathrm{Et}_{2} \mathrm{O}$ & 0.0 & 0.47 & 0.27 & 0.0 & 3.89 \\
\hline Dioxane & 0.0 & 0.37 & 0.55 & 0.0 & 2.19 \\
\hline Acetone & 0.07 & 0.48 & 0.72 & 0.0 & 19.75 \\
\hline DMSO & 0.0 & 0.76 & 1.0 & 0.0 & 45.80 \\
\hline $\mathrm{CHCl}_{3}$ & 0.34 & 0.0 & 0.76 & 0.5 & 4.55 \\
\hline $\mathrm{CH}_{2} \mathrm{Cl}_{2}$ & 0.22 & 0.0 & 0.80 & 0.5 & 8.54 \\
\hline $\mathrm{MeCN}$ & 0.07 & 0.48 & 0.72 & 0.0 & 36.60 \\
\hline EtOH & 0.86 & 0.77 & 0.54 & 0.0 & 24.20 \\
\hline $\mathrm{MeOH}$ & 0.98 & 0.62 & 0.60 & 0.0 & 30.71 \\
\hline $\mathrm{H}_{2} \mathrm{O}$ & 1.13 & 0.18 & 1.09 & 0.0 & 76.70 \\
\hline $\mathrm{CF}_{3} \mathrm{CH}_{2} \mathrm{OH}$ & 1.51 & 0.0 & 1.02 & 0.0 & - \\
\hline
\end{tabular}

${ }^{a}$ The dielectric constants are reported for a temperature of $35^{\circ} \mathrm{C}$ as calculated from the data given in Ref. (10).

${ }^{b}$ Values of 0.0 or 0.1 may bc used for this parameter. By using both we find that better results are obtained when 0.0 is used in all cases considered here.

ing to the oxygen atom of pyridine $N$-oxide is large, since the magnitude of the $a$ term is comparable to that observed for pyridine. Thus hydrogen bonding to a neighboring oxygen atom can produce nitrogen shielding changes comparable to those found when nitrogen is directly involved in hydrogen bonding. This is the first positive demonstration of this effect. Consequently care is required in interpreting large nitrogen shielding increases, as a function of solvent.

The term $S$, which includes polarizability-polarity effects, is also of interest. Our present results indicate that the influence of these effects on the nitrogen shielding in both pyridine $N$-oxide and pyridine is comparable in sign and magnitude, such that an increase in polarity of the solvent produces a nitrogen shielding increase for both molecules. This is opposite to that observed for nitrogen atoms in nitro moieties, for example, nitromethane and methyl nitrate $(5,6)$.

Solvaton calculations, using the INDO/S-SOS procedure (7), are reported for the nitrogen shielding of pyridine $N$-oxide in Table 4 . These results support both the sign and the magnitude of the effects of the solvent polarizability-polarity interaction of pyridine $\mathrm{N}$-oxide. Comparison with the results of solvaton calculations on nitro moieties also shows a good agreement with the observed nitrogen shielding trends $(5,6)$.

The term involving the parameter $d$ in Eq. [1] is fairly small but the term containing $b$, which describes the response of the nitrogen shielding to the hydrogen bond acceptor strength of the solvent, is significant for pyridine $N$-oxide. This appears to indicate that some weak interactions occur between the hydrogen atoms of pyridine 
TABLE 3

Results of a Least-Squares Fitting ${ }^{a}$ of the Nitrogen Shielding of Pyridine $\mathrm{N}$-Oxide in a Variety of Solvents to Eq. [1]

\begin{tabular}{lcc}
\hline \multicolumn{1}{c}{ Solvent } & $\begin{array}{c}\text { Experimental } \\
\text { shielding }\end{array}$ & $\begin{array}{c}\text { Shielding predicted } \\
\text { by Eq. [1] }\end{array}$ \\
\hline Cyclohexane & 76.47 & 76.56 \\
$\mathrm{Et}_{2} \mathrm{O}$ & 78.78 & 79.86 \\
$\mathrm{CCl}_{4}$ & 80.84 & 79.23 \\
$\mathrm{Benzene}_{\text {Dioxane }}$ & 81.36 & 81.94 \\
Acetone & 81.40 & 81.02 \\
$\mathrm{DMSO}_{\mathrm{MH}} \mathrm{Cl}{ }_{2}$ & 82.85 & 83.51 \\
$\mathrm{CHCl}_{3}$ & 85.64 & 85.01 \\
$\mathrm{EtOH}$ & 85.66 & 85.59 \\
$\mathrm{MeOH}$ & 86.78 & 87.30 \\
$\mathrm{CF}_{3} \mathrm{CH}_{2} \mathrm{OH}$ & 97.06 & 96.37 \\
$X Y Z_{0}=76.57 \pm 0.74$ ppm & 98.05 \\
$a=16.11 \pm 0.67$ ppm per unit value of $\alpha$ & 106.53 \\
$b=3.85 \pm 1.17$ ppm per unit value of $\beta$ \\
$S=5.52 \pm 1.11$ ppm per unit value of $\pi^{*}$ \\
$d=0.38 \pm 0.26$ (dimensionless) \\
\hline
\end{tabular}

${ }^{a}$ In the fitting procedure the standard deviation is $0.94 \mathrm{ppm}$ and the correlation coefficient is 0.99 .

$N$-oxide and the hydrogen bond acceptor sites of the chosen solvents. Similar observations have previously been made for a variety of structures containing electron attracting groups $(5,6)$.

\section{TABLE 4}

INDO/S-SOS Shieldings ${ }^{a}$ Calculated Using the Solvaton Model for Pyridine $N$-Oxide, Expressed with Respect to That of the Molecule in a Solution of Dielectric Constant of $2^{b}$

\begin{tabular}{cc}
\hline Dielectric constant $\epsilon$ & Variation of N shielding \\
\hline 2 & $0.0^{c}$ \\
4 & 2.52 \\
10 & 3.37 \\
20 & 3.55 \\
40 & 3.61 \\
\hline
\end{tabular}

${ }^{a}$ All shieldings are in ppm.

${ }^{b}$ The value of $\epsilon-2$ is arbitrarily chosen as the origin to correspond to values of $\epsilon$ for a number of hydrocarbon solvents.

'Taken as arbitrary zero. 
As a result of this study we conclude that hydrogen bonding to atoms which are in close vicinity to nitrogen atoms can also produce remarkable nitrogen shielding effects. These effects are similar in both sign and magnitude to those observed when the nitrogen atom is directly involved in hydrogen bonding. Such effects on the shielding can be larger than those produced by medium polarity effects. The latter effects on nitrogenous moieties, where the nitrogen bears a formal positive charge, can produce shielding changes which vary in sign. However, in aromatic $N$-oxides the medium polarity effects on the nitrogen shielding appear to be of the same sign as those in the parent heterocycle.

\section{EXPERIMENTAL}

Since pyridine $N$-oxide is a hygroscopic substance which forms stable hydrates particular attention was paid to dried samples and solvents. Pyridine $\mathrm{N}$-oxide was dried in vacuum over $\mathrm{P}_{2} \mathrm{O}_{5}$ and distilled under reduced pressure. Where applicable the solvents were dried. The alcohols used were distilled over magnesium, and the chlorinated solvents were passed through a column of basic alumna directly before use. DMSO was distilled over calcium hydride, benzene was distilled over $\mathrm{P}_{2} \mathrm{O}_{5}$, and diethyl ether was distilled over sodium. All sample preparations were performed in an atmosphere of dry argon.

The nitrogen shielding results were produced by high-precision ${ }^{14} \mathrm{~N}$ PFT NMR measurements at a field which gives the signal of our primary standard, neat nitromethane, at a frequency of $36.141524 \mathrm{MHz}$. As discussed elsewhere (12) this field produces the resonance of a bare nitrogen nucleus at $36.136826 \mathrm{MHz}$. This frequency is used in obtaining the reported nitrogen shieldings with respect to neat nitromethane. All measurements were taken at $35.0 \pm 0.2^{\circ} \mathrm{C}$ which is maintained by a VT unit on a Bruker AM500 instrument; concentric tubes, $10 / 4 \mathrm{~mm}$, were used and in the inner tube was a $0.3 \mathrm{M}$ solution of nitromethane in acetone- $d_{6}$ which provides both a deuterium lock and our secondary standard. The sample to be studied was contained in the outer tube. The nitrogen shielding of the $0.3 \mathrm{M}$ solution of nitromethane has been measured, by means of spherical concentric containers in order to remove bulk susceptibility effects on nitrogen shielding, and is found to be $0.77 \mathrm{ppm}$ (4) with respect to that of neat nitromethane. The shieldings presented in Table 1 are given with respect to that of neat nitromethane after including bulk susceptibility corrections and $0.77 \mathrm{ppm}$ for the standard used as described by

$$
\sigma_{\mathrm{N}}(\text { ref I })=\sigma_{\mathrm{N}}(\operatorname{ref} \text { II })+0.77-\frac{1}{3}\left(\chi_{\text {ref II }}-\chi_{\text {sample }}\right),
$$

where ref $\mathrm{I}$ is neat liquid nitromethane, ref II is a $0.3 \mathrm{M}$ solution of nitromethane in acetone- $d_{6}$, and $\chi$ is the volume magnetic susceptibility in SI units (12). The measurements were taken with the sample and reference tubes in parallel to the applied magnetic field. The following parameters were commonly used in the ${ }^{14} \mathrm{~N}$ measurements: $90^{\circ}$ pulse corresponding to $40 \mu \mathrm{s}$; spectral width about $8 \mathrm{kHz}$ with quadrature detection; acquisition time about $0.13 \mathrm{~s}$; zero relaxation delay; and about 2000 accumulated scans per spectrum. No exponential multiplication of the FID was employed before the FT. The ${ }^{14} \mathrm{~N}$ peak positions were obtained by means of a Lorentzian lineshape fitting procedure applied independently to the nitromethane signal and to that 
of the sample concerned. The frequency obtained for the sample is subtracted from that of nitromethane and the difference divided by the frequency of a bare nitrogen nucleus to give the relative nitrogen shielding of the sample. The signal fitting procedure also produced the ${ }^{14} \mathrm{~N}$ linewidths.

The INDO/S-SOS solvaton shielding calculations were performed on the University of Surrey Primenet system using procedures given elsewhere (7); standard geometries were used in the calculations (13).

\section{ACKNOWLEDGMENT}

We are grateful for funding from Project CPBP 01 13.1.25 of the Polish Academy of Sciences.

\section{REFERENCES}

1. M. Witanowski, L. STEFANiak, AND G. A. WebB, "Annual Reports on NMR Spectroscopy" (G. A. Webb, Ed.), Vol. 18, pp. 81-85, Academic Press, London, 1986.

2. M. WITANOWSKI, L. StefaniaK, AND G. A. WebB, "Annual Reports on NMR Spectroscopy" (G. A. Webb, Ed.), Vol. 11B, p. 35, Academic Press, London, 1981.

3. G. A. WEBb AND M. WITANOWSKI, Proc. Indian Acad. Sci. 94, 241 ( 1985).

4. M. Witanowski, L. Stefaniak, B. Na Lamphun, And G. A. WebB, Org. Magn. Reson. 15, 57 (1981).

5. M. Witanowski, T. Sitkowski, S. Biernat, B. Kamienski, B. T. Hamdi, and G. A. WebB, Magn. Reson. Chem. 23, 748 (1985).

6. M. WitAnOwski, T. SitKowski, S. BIERnAt, L. V. Sudha, AND G. A. WebB, Magn. Reson. Chem. 25, 725 (1987).

7. I. ANDO AND G. A. WeBB, "Theory of NMR Parameters," Academic Press, London, 1983.

8. G. KuopMan, Chem. Phys. Lett. 1, 200 (1967).

9. M. J. Kamlet, J. L. M. AbBoud, And R. W. Taft, Prog. Phys. Org. Chem. 13, 485 (1980).

10. R. C. Weast (Ed.), "Handbook of Chemistry and Physics," 64th ed., p. E-49, Chemical Rubber Co., Cleveland, Ohio, 1984.

11. M. WitANowski, L. StefaniaK, AND G. A. Webb, "Annual Reports on NMR Spectroscopy" (G. A. Webb, Ed.), Vol. 18, pp. 70-77, Academic Press, London, 1986.

12. M. WITANOWSKI, L. STEFANiAK, AND G. A. WEBB, “Annual Reports on NMR Spectroscopy" (G. A. Webb, Ed.), Vol. 18, pp. 17-30 and 218-221, Academic Press, London, 1986.

13. J. A. Pople AND M. S. Gordon, J. Am. Chem. Soc. 89, 4233 (1967). 